Der intelligente Fußboden

\title{
Verhaltensmuster bei Demenz erkennen
}

— Das Verhalten Demenzkranker ist mit ausgeprägten Risiken verbunden. Insbesondere die Sturzgefahr und Orientierungslosigkeit sowie das Fluchtrisiko ist bei Demenzkranken stark erhöht. Der finnische Hersteller MariCare Oy hat mit dem Fußbodensystem Elsi ${ }^{\circledR}$ Smart Floor eine Technologie entwickelt, welche unsichtbar und unkompliziert in Pflegeheimen oder zu Hause eingebaut werden kann. Hierüber können Stürze oder andere ungewöhnliche Bewegungsabläufe erkannt werden.

Als umfassendes Assistenzsystem stellt der intelligente Fußboden Funktionalitäten bereit, die den größten Teil der täglichen Risiken vermeiden oder reduzieren helfen. Er kann das Pflegepersonal auf Folgen vieler verschiedener Demenzsymptome und Verhaltensstörungen aufmerksam machen. Ob ein Patient schläft, isst, sein Zimmer verlässt oder die Toilette aufsucht - das Pflegepersonal wird darauf aufmerksam gemacht und kann eine Datenbasis aufbau- en und ein Bewegungsmuster definieren. Der Einbau des Fußbodens kann Verletzungen reduzieren und eine vorausschauende Pflege durch schnellere Reaktion verbessern. Ausgehend von den Symptomen und Bedürfnissen jedes einzelnen Heimbewohners können die Mitarbeiter ihre Aufgaben so weitaus effektiver planen.

Diese Technologie nutzt kapazitive und induktive Sensorik, ähnlich einem Touchpad oder Sensorbildschirm. Das Vorhandensein des Systems ist optisch nicht wahrnehmbar. Die Heimbewohner merken nicht, dass sie überwacht werden, sodass normalerweise keine Probleme durch Interaktion mit dem Assistenzsystem entstehen, die dessen Funktion behindern könnten.

Der Einbau ist schnell und einfach, ähnlich einer Bodenunterlage. Das System wird durch den normalen Bodenbelag geschützt und ist daher langlebig.

www.maricare.com
Mobiler Hausnotruf Notrufarmband für Senioren

— Mit dem Zembro Notrufarmband können Senioren im Ernstfall überall zu jeder Zeit Hilfe rufen. Zuvor festgelegte Notfallkontakte werden dann über die ZembroApp alarmiert. Sie können den Standort des Trägers in der App einsehen und direkt auf dem Armband anrufen. Das neue Zembro Plus widmet sich nun der Betreuung von Demenz- oder Alzheimerkranken. Das Notrufarmband besitzt einen einzigartigen $\mathrm{Si}$ cherheitsverschluss, der nur mit Hilfe einer zweiten Person geöffnet werden kann. So wird verhindert, dass Demenzpatienten das Armband selbstständig ablegen. Durch GPS-Ortung kann außerdem eine virtuelle Grenze um ein festgelegtes Gebiet gezogen werden. Sobald der Träger des Notrufarmbands diesen sicheren Bereich verlässt, wird ein Alarm ausgelöst und die Notfallkontakte werden automatisch kontaktiert.

www.zembro.com

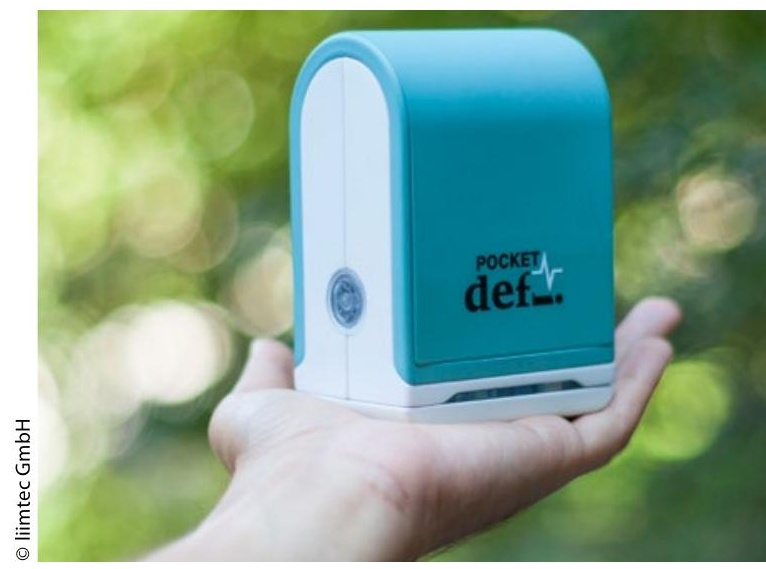

fert wird er ab Dezember 2018, vorausgesetzt, dass die Kampagne auf Indiegogo die für die Zulassung benötigten finanziellen Mittel erzielt. Die ersten Interessenten können einen Preisvorteil von über $40 \%$ nutzen und zahlen für das Einführungspaket mit einem Basisgerät, einem Set Elektroden und einer Ladeplatte sowie der App ab 399 Euro.

www.liimtec.at 